\title{
Beef with transgenic fish
}

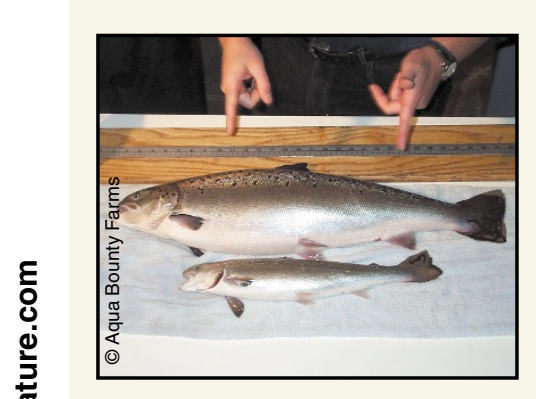

Led by US public interest group the Center for Food Safety (Washington, DC), more than 60 consumer and environmental groups, along with fishing companies and fishermen, filed a petition to the US FDA on May 9 requesting "a moratorium on the domestic marketing, importation, and exportation of transgenic fish. . .until the FDA establishes a comprehensive framework. . .to fully address the human health and environmental impacts caused by the commercialization of transgenic fish." There are currently about 40 transgenic fish being developed worldwide, but the focus has been on transgenic Atlantic salmon developed by A/F Protein (Waltham, MA) that reach commercial size in half the time as wild type, but need only about $80 \%$ of the food to do so (Nat. Biotechnol. 18, 143, 2000). The salmon is currently under review as a drug by the FDA's Center for Veterinary Medicine, which collaborates closely with other federal and state agencies particularly on environmental matters. "They're demanding a moratorium and that the FDA has to first approve [transgenic fish] before they're allowed into the system, and that's exactly what [the FDA is] doing anyway," asserts Elliot Entis of A/F. "Where's the beef?"

$A B$

\section{Lupus drug reviewed}

On April 19, the FDA's Arthritis Advisory Committee discussed the fate of Aslera, Genelabs Technologies' (Redwood City, CA) synthetic dehydroepiandrosterone (DHEA) treatment for systemic lupus erythematosus. The meeting largely pits clinicians, who are keenly aware of the void of regulated therapies for lupus, against an FDA statistical review that repeatedly characterized Genelabs' data as "not statistically significant," particularly for adverse events when compared with placebo. Despite this criticism, Genelabs' vice president of finance Matthew Loar argues that these were the first well-controlled studies in lupus, for which there has been a long-standing lack of consensus on how to judge efficacy (Nat. Biotechnol. 17, $319,1999)$. In an unusual move, the FDA did not ask the Arthritis Advisory Committee to vote; without a formal recommendation, it promised to render its decision on DHEA, which has been granted orphan status, by June 26. DHEA has long been sold as an unregulated dietary supplement.

\section{Merck buys Rosetta}

Pharmaceutical giant Merck (Whitehouse Station, NJ) acquired Rosetta Inpharmatics (Kirkland, WA) on May 11 in an all-share deal valued at approximately $\$ 620$ million. Rosetta, whose main source of income comes from bioinformatics collaborations, will operate as an independent subsidiary and continue to preserve the confidentiality of research deals with current customers. UBS Warburg (New York) analyst Michael Clulow feels that this acquisition validates an IT approach to drug discovery: "I think you're going to see a lot of other pharmaceutical companies stand up and say 'we need to stake an investment in IT." Clulow points to Bayer's (Leverkeusen, Germany) multi-million dollar collaborations with bioinformatics companies Lion (Heidelberg, Germany) and Curagen (New Haven, CT) (Nat. Biotechnol. 19, 186, 2001). "What's the obstacle of Bayer deciding to come in and take those companies over?" Rosetta, with $\$ 150$ million in the bank-enough to last "indefinitely," according to Culow-jumped $75 \%$ to $\$ 17.34$ the day of the news.

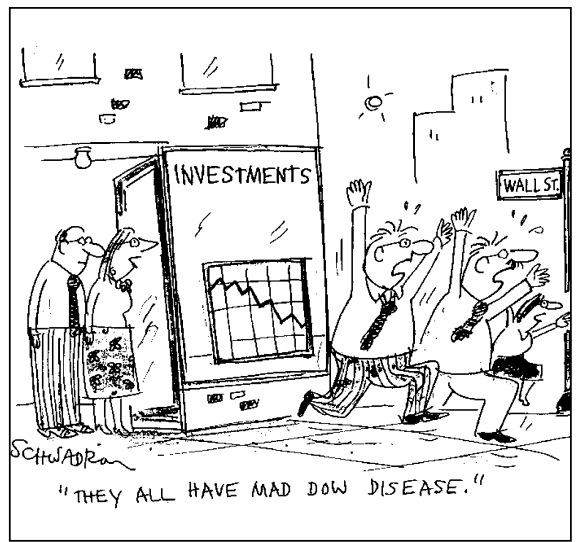

Biomira, Merck in vaccine deal

German pharmaceutical company Merck (Darmstaad) has expanded its oncology pipeline through a co-development and marketing agreement with Biomira (Edmonton, Canada). Biomira could receive up to $\$ 150$ million in up-front license fees, milestones, and equity for rights to two vaccines: Theratope, in phase III metastatic breast cancer trials, and BLP25, for non-small cell lung cancer (phase II). The vaccines boost the body's immune system against cancers by mimicking cancer-specific cell surface glycoproteins. A spokesperson for Biomira says that if Theratope is successful, not only could it become the first vaccine approved for cancer, but also its indication could be extended to include earlier stages of breast cancer and other solid tumors. In theory, Theratope could be effective in more patients than Genentech's (S. San Francisco, CA) and Roche's (Basel, Switzerland) Herceptin, which is effective in only a third of breast cancer patients. The vaccines fit well within Merck's growing portfolio of novel immunotherapeutic approaches to treating cancer, including antibodies, angiogenesis inhibitors, and cytokines. Biomira will share the cost of clinical development, but will also share profits on sales in North America and receive royalties on sales elsewhere. $\quad L F$

\section{New Product Approvals \\ Product}

Companies

Details

Hemopure

(bovine hemoglobin glutamer)
Biopure (Cambridge, MA) Tshepo Pharmaceuticals (Sandton, S. Africa)
On April 10, South Africa approved this oxygen therapeutic for acute anemia and low levels of red blood cells in adult surgery patients. Hemoglobin is purified from US certified disease-free cattle, and then chemically cross-linked for stability. Tshepo will begin marketing Hemopure in S. Africa in 2002, until which time Biopure will provide the product for free. Biopure intends to file for approval in the United States and Europe later this year. 